\title{
Factors Affecting Detection of Brucella melitensis by BACTEC NR730, a Nonradiometric System for Hemocultures
}

\author{
CARLOS GAMAZO, ${ }^{1 *}$ ANA I. VITAS, ${ }^{1}$ IGNACIO LÓPEZ-GOÑI, ${ }^{1}$ RAMÓN DÍAZ, ${ }^{1,2}$ \\ AND IGNACIO MORIYÓN ${ }^{1}$ \\ Departamento de Microbiología, Facultad de Medicina, ${ }^{1}$ and Departamento de Microbiología, \\ Clínica Universitaria, ${ }^{2}$ Universidad de Navarra, Apartado 273, 31080 Pamplona, Spain
}

Received 6 July 1993/Returned for modification 27 August 1993/Accepted 8 September 1993

\begin{abstract}
The detection of Brucella bacteremia by subculture does not always correlate with a positive signal in the BACTEC NR730 nonradiometric system (Becton Dickinson Diagnostic Instrument Systems, Sparks, Md.). The effect of the inoculum size, $\mathrm{pH}$, sodium polyanetholesulfonate, carbon sources ( $i$-erythritol, sodium pyruvate, monosodium glutamate, D-glucose, and $\mathrm{L}$-alanine), and urea in the release of $\mathrm{CO}_{2}$ was evaluated by using the reference strain Brucella melitensis 16M. In standard NR6 vials with or without blood, inocula 5 to 10 times larger (at least 265 CFU per vial) than those usually found in the blood of patients with brucellosis were necessary to produce a positive growth value (GV) in 4 days or less, and similar results were obtained with vials supplemented with the substrates listed above. GVs were consistently lower in vials with sodium polyanetholesulfonate than in vials without this agent. Vials with no blood inoculated with 265 CFU per vial showed turbidity 1 day before GVs became positive, proving that the major limiting detection factor was the low level of release of $\mathrm{CO}_{2}$ and not an inadequate growth medium. In NR6 vials bufiered to pH 6.2, GVs became positive faster and were higher than those in standard vials. NR6 vials at $\mathrm{pH} 6.2$ with $0.3 \%$ sodium pyruvate yielded a positive GV in the first day of bacterial turbidity.
\end{abstract}

The diagnosis of human brucellosis is based on microbiological and serological laboratory tests. The presence of antibodies does not always mean an active case of brucellosis, and therefore, serological results must be interpreted in light of clinical and bacteriological data. The unequivocal proof of an active Brucella infection is the culture, isolation, and identification of the causative microorganism, and the blood broth culture under a $10 \% \mathrm{CO}_{2}$ atmosphere is the simplest and most often used procedure (6). However, conventional blood cultures for Brucella spp. present several problems. First, unless Castañeda's method (1) is adopted, a broth culture suspected of containing Brucella spp. must be streaked out onto a solid medium, and when performed routinely, this step represents a real risk of infection for laboratory personnel (12). Moreover, since the majority of conventional Castañeda blood cultures for Brucella spp. are positive between days 7 and 21 and $2 \%$ are positive after day 27 (23), long incubation periods are necessary before a blood culture can be rejected as negative for Brucella spp. Several automated methods for the fast detection of bacteremias have been described. These methods avoid unnecessary sampling and streaking out of blood cultures. The semiautomatic nonradiometric system for hemocultures, BACTEC NR730 (Becton Dickinson Diagnostic Instrument Systems, Sparks, Md.), is one of the most widely used. This system detects bacterial growth by infrared spectroscopy measurement of the release of $\mathrm{CO}_{2}$. Although the BACTEC NR730 system is more rapid and reliable than other systems $(5,11$, 31), it presents problems such as false-negative results or the delayed detection of bacteremias caused by Neisseria, Candida, or Streptococcus spp. $(2,24,33)$. Moreover, Serrano et al. (25) found that protracted periods of incubation are

\footnotetext{
* Corresponding author.
}

necessary to detect Brucella melitensis with the BACTEC NR730 system. Several reasons could explain this observation. First, the bacterial concentration found in individuals with Brucella bacteremia is usually low. Second, the medium used in BACTEC vials could be unsuitable for Brucella growth. Finally, because of the peculiarities of Brucella metabolism $(21,22)$, the $\mathrm{CO}_{2}$ release could be not enough to be detectable.

In the work described here, we studied the influence of these factors for the prompt detection of Brucella spp. by the BACTEC NR730 system using the reference strain $B$. melitensis $16 \mathrm{M}$.

\section{MATERIALS AND METHODS}

Experimental design. Aerobic BACTEC NR6A (NR6) and BACTEC NR6AX (NR6AX) vials were used; both were supplied by the manufacturer (Becton Dickinson-Spain, Madrid, Spain). The components of the NR6 vial were tryptic soy broth $(2.75 \%)$, hemin $(0.0005 \%)$, vitamin $K$ $(0.00005 \%)$, glucose $(0.06 \%)$, sucrose $(0.0835 \%)$, sodium polyanetholesulfonate (SPS) $(0.025 \%)$, yeast extract $(0.25 \%)$, and pyridoxal $\mathrm{HCl}(0.001 \%)$ in $30 \mathrm{ml}$ of purified water $(\mathrm{pH} \mathrm{7.2)}$. The composition of NR6AX broth was that of NR6 but without SPS. Various organic substrates and pHs in NR6 vials were tested individually or in different combinations (see below). When used, blood was obtained from healthy donors, and blood was added ( 3 to $4 \mathrm{ml}$ per vial) immediately after extraction. The sterility of the blood samples was examined for each experiment by adding the same volume to noninoculated vials.

The organism used was the virulent strain $B$. melitensis 16M ATCC 12345 (reference strain of biotype 1). To ensure the smoothness of the strain, it was inoculated intraperitoneally into mice, recovered from the spleen 2 weeks later, 
and tested for dissociation (1). The fresh isolate was used to prepare a stock suspension $\left(3.6 \times 10^{7} \mathrm{CFU} / \mathrm{ml}\right)$ in skim milk that was stored at $-70^{\circ} \mathrm{C}$. The inocula were prepared by appropriate dilution of the stock suspension in sterile saline. For each experiment, the actual size of the inoculum was measured by spreading triplicate $0.1-\mathrm{ml}$ samples of the inoculum onto tryptic soy agar plates and incubating the plates for 4 days at $36^{\circ} \mathrm{C}$.

Vials were incubated at $36^{\circ} \mathrm{C}$ with constant shaking (Orbital Shaker 650; Becton Dickinson Diagnostic Instrument Systems, Towson, Md.), and when turbidity was apparent (1.0 on the McFarland scale in vials without blood), growth values (GVs) were recorded with the BACTEC system. Following the manufacturer's instructions, a GV of $\geq 20$ or $\geq 35$ was considered positive for vials without or with blood, respectively. All the experiments were performed at least twice by using 5 to 10 vials per experiment. To counteract the effects of $\mathrm{pH}$ and blood per se on the release of $\mathrm{CO}_{2}$, uninoculated control vials at different $\mathrm{pHs}$ (see below) with and without blood supplement were included in all the experiments. Their GVs were subtracted from the ones obtained in the respective inoculated vials.

Carbon sources. The supplements and the range of final concentrations were as follows: sodium pyruvate, 3 to 12 $\mathrm{mg} / \mathrm{ml}$; monosodium glutamate, 2.5 to $10.0 \mathrm{mg} / \mathrm{ml} ; i$-erythritol, 0.05 to $2.00 \mathrm{mg} / \mathrm{ml}$; urea, 2 to $20 \mathrm{mg} / \mathrm{ml}$; D-glucose, 2.5 to $10.0 \mathrm{mg} / \mathrm{ml}$; and $\mathrm{L}$-alanine, 3 to $12 \mathrm{mg} / \mathrm{ml}$. All supplements were purchased from Sigma Chemical Co. (St. Louis, Mo.). Working solutions were sterilized by filtration $(0.20-\mu \mathrm{m}$ pore size; DynaGard-ME; Microgon Inc., Laguna Hills, Calif.) and were added aseptically to the BACTEC vial. Noninoculated vials were used to verify the sterilities of the solutions.

pH adjustment. Different proportions of $0.5 \mathrm{M} \mathrm{KH}_{2} \mathrm{PO}$ and $0.5 \mathrm{M} \mathrm{Na}_{2} \mathrm{HPO}_{4}$ were added aseptically to the BACTEC vials to adjust the $\mathrm{pH}$ in the range of 6.0 to 7.2 (4). By this method, the maximum concentration of total phosphorus in the vials was $0.30 \%$ for $\mathrm{pH} 6.0$, which is lower than the inhibitory concentration for Brucella spp. $(0.70 \%$ [35]). This method resulted in a stable $\mathrm{pH}$ throughout the incubation periods, as shown by measurements performed in noninoculated vials and in inoculated vials taken at different incubation times and sterilized by autoclaving.

\section{RESULTS}

Effect of inoculum size. Figure 1 shows the influence of the inoculum size on the GV in NR6 vials (no blood). Bacterial turbidity was apparent on the third day with the highest inoculum $(2,650$ CFU per vial) and on the fourth day with the other inocula. However, with $265 \mathrm{CFU}$ per vial or greater, a positive GV signal was obtained on the fourth day, while vials with lower inocula were negative until the sixth day of incubation. Since smaller inocula are found in patients with Brucella bacteremia, 15 to 45 CFU per vial (equivalent to a final concentration of approximately $1 \mathrm{CFU} / \mathrm{ml}$ ) was used in subsequent experiments.

Efiect of SPS. NR6 and NR6AX vials (with and without SPS, respectively) inoculated with the same amount of bacteria showed the same turbidity $(1.0$ on the McFarland scale) after 4 days of incubation, and both had a negative GV. However, NR6AX vials had a positive GV (mean \pm standard deviation, $27.3 \pm 16.3$ ) on the fifth day of incubation, while NR6 vials were still negative $(\mathrm{GV}, 10.1 \pm 4.9)$ (Fig. 2). Similar results were observed when blood supple-

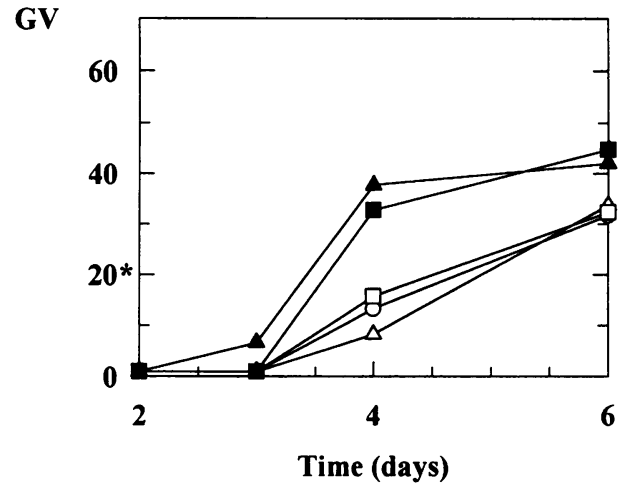

FIG. 1. Effect of inoculum size on the GV in NR6 vials without blood. The following inoculum sizes (CFU per vial) were used: 3 $(\triangle), 10(O), 33(\square), 265(\square)$, and 2,650( $\triangle)$. The asterisk indicates the positive GV threshold.

ment was used: $36.8 \pm 11.9$ in NR6AX vials versus $18.2 \pm$ 1.7 in NR6 vials on the fifth day of incubation.

Efiect of carbon sources in standard vials at pH 7.2. The addition of any of the carbon substrates to standard NR6 vials did not generate a positive GV in the first day of bacterial turbidity (fourth day of incubation). A small but consistent increase in the GV was observed only in the vials supplemented with 0.6 or $1.2 \%$ L-alanine on the sixth day of incubation (Fig. 3, $\mathrm{pH} 7.2$ ). The addition of $0.6 \%$ sodium pyruvate also caused a small increase in the GV, although this did not occur consistently (data not shown).

Effect of pH, L-alanine, and sodium pyruvate. In standard NR6 vials, the $\mathrm{pH}$ changed from 7.2 to 6.9 upon inoculation and incubation for up to 6 days. When the $\mathrm{pH}$ of the nonsupplemented NR6 broth was adjusted to 6.2 (Fig. 3 and 4), $\mathrm{CO}_{2}$ release was significantly increased, with a positive GV on the fifth day of incubation (inoculum of 19 CFU per vial). However, on the fifth day, vials with $\mathrm{pHs}$ of greater than 6.2 still gave a negative GV (Fig. 4). In addition, the bacterial turbidity in vials at $\mathrm{pH} 6.2$ was apparent $7 \mathrm{~h}$ before bacterial turbidity was apparent in vials at $\mathrm{pH}$ 7.2. Moreover, at $\mathrm{pH} 6.2$ both alanine and pyruvate had a marked effect on the GV. Vials at $\mathrm{pH} 6.2$ supplemented with $0.6 \%$ alanine gave a positive GV on the fourth day of incubation (Fig. 3), and with $0.6 \%$ sodium pyruvate, even higher GVs were consistently observed (Fig. 4). The addition of this

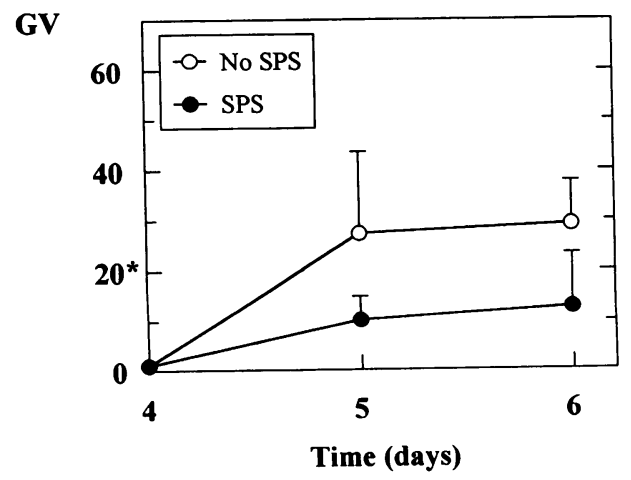

FIG. 2. Effect of SPS on the GV of B. melitensis 16M. NR6 vials (with SPS) and NR6AX vials (without SPS) were inoculated with 16 CFU per vial. The asterisk indicates the positive GV threshold. 


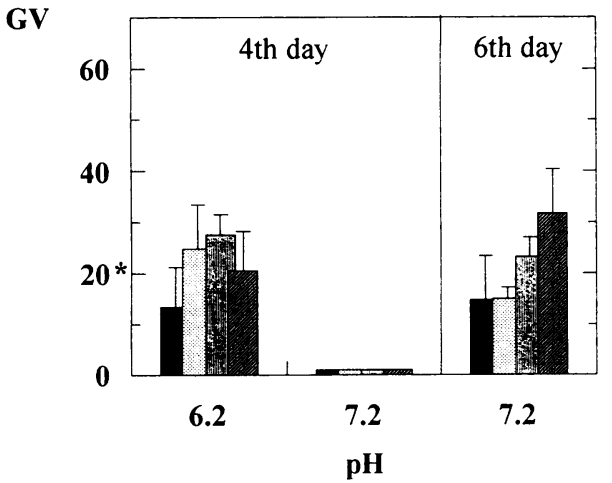

FIG. 3. Effect of $\mathrm{pH}$ and L-alanine on the GV. NR6 vials were supplemented with 0.3 (固), 0.6 (圆), or $1.2 \%$ (四) L-alanine, and the $\mathrm{pH}$ was adjusted to 6.2 or 7.2. The inoculum used was $42 \mathrm{CFU}$ of $B$. melitensis $16 \mathrm{M}$ per vial. Standard vials $(\square)$ were used as controls. The asterisk indicates the positive GV threshold.

compound in vials at $\mathrm{pH} 6.6$ increased the $\mathrm{GV}$ on the fifth day of incubation (Fig. 4). Furthermore, with the pyruvate supplement, turbidity appeared 5 and $12 \mathrm{~h}$ before turbidity appeared in vials without pyruvate at $\mathrm{pH} 6.2$ and in standard vials, respectively. Similar results were obtained in experiments performed with blood in the vials: a positive GV $(70.0$ \pm 7.8 ) in vials with $0.6 \%$ sodium pyruvate at $\mathrm{pH} 6.2$ on the fifth day of incubation, when standard vials were still negative.

\section{DISCUSSION}

The BACTEC NR730 is a semiautomatic system that is based on the detection of the $\mathrm{CO}_{2}$ released during bacterial growth. It was created for the fast and safe detection of bacteremia. Our results show that the release of $\mathrm{CO}_{2}$ by $B$. melitensis was not high enough to be detected as positive by the system and that it was clearly preceded by Brucella growth. This observation is consistent with the low yield of $\mathrm{CO}_{2}$ per hydrocarbon molecule assimilated reported for Brucella abortus (10) and explains the delayed detection of Brucella bacteremia with the BACTEC system compared with that by conventional subculturing (25).

Several factors can account for that delay: first, the low concentration of bacteria usually found in patients with
Brucella bacteremia (1 to $5 \mathrm{CFU} / \mathrm{ml}$ [17]) and the comparatively long $(2.5$ to $3.5 \mathrm{~h}[14,20])$ doubling time of the genus. In our experiments, only inocula of $265 \mathrm{CFU}$ per vial or higher generated a positive GV after 4 days of incubation. A similar influence of the size of the inoculum has been reported by Zimmerman et al. (34). Second, in contrast to most aerobic bacteria that channel glucose into the Krebs cycle through the Embden-Meyerhof-Parnas and EntnerDoudoroff pathways, both radiorespirometric (21) and enzymatic (22) studies support a model for Brucella spp. in which the bulk of glucose catabolism is via the hexose monophosphate pathway. Thus, substrates that bypass this pathway and that are fed directly into the Krebs cycle could enhance $\mathrm{CO}_{2}$ release, a hypothesis supported by our observation that both alanine and pyruvate accelerated $B$. melitensis $16 \mathrm{M}$ growth and increased $\mathrm{CO}_{2}$ release. The other substrates tested could not be incorporated directly into the Krebs cycle (erythritol [26, 27]), or their oxidation (glutamate) could be affected by the cell's permeability (32). Although $B$. melitensis often presents a strong urease activity, the addition of this substrate did not increase the release of $\mathrm{CO}_{2}$. Finally, since the solubility of $\mathrm{CO}_{2}$ in the broth is affected by the $\mathrm{pH}$, the release of $\mathrm{CO}_{2}$ into the vial's atmosphere should be lessened in bacteria that, like Brucella spp., do not acidify the growth medium. We found that reduction of the $\mathrm{pH}$ from 7.2 to 6.2 was enough to generate a positive GV signal in the first day of bacterial growth. Indeed, the combination of sodium pyruvate and low $\mathrm{pH}$ produced a further increase in $\mathrm{CO}_{2}$ release, as observed before by several investigators $(8$, 21).

The modifications introduced into the NR6 broth should not affect the aerobic bacteria that have classical glucose metabolic pathways and that are found in blood cultures. In fact, supplementation of the NR6 vials with $0.6 \%$ sodium pyruvate at $\mathrm{pH} 6.2$ generated a higher $\mathrm{GV}$ than that in standard vials (100 CFU per vial) of clinical isolates of Staphylococcus aureus, Staphylococcus epidermidis, Salmonella enteritidis, Salmonella typhimurium, Citrobacter freundii, Proteus mirabilis, Streptococcus faecalis, Klebsiella pneumoniae, and Acinetobacter calcoaceticus (30).

Although the addition of SPS increases the growth of most bacteria in hemocultures, it has been reported that it has an adverse effect on the growth of Neisseria meningitidis, Neisseria gonorrhoeae, Peptostreptococcus anaerobius, Streptobacillus moniliformis, and Gardnerella vaginalis (7, $9,13,18,24,28)$. The same negative effect was observed for
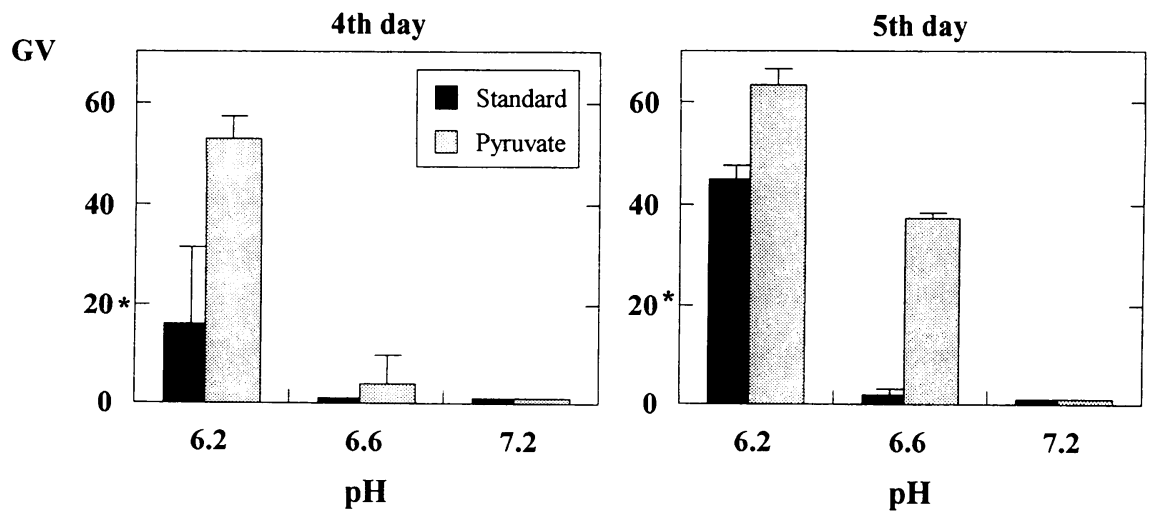

FIG. 4. Effect of $\mathrm{pH}$ and sodium pyruvate on the GV. NR6 vials were supplemented with $0.6 \%$ sodium pyruvate, and the $\mathrm{pH}$ was adjusted to $6.2,6.6$, or 7.2 . The inoculum used was $19 \mathrm{CFU}$ of $B$. melitensis $16 \mathrm{M}$ per vial. Standard vials were used as controls. The asterisk indicates the positive GV threshold. 
B. melitensis $16 \mathrm{M}$, since GVs were higher in vials without SPS (NR6AX) than in vials with SPS (NR6). This adverse effect of SPS could be due to the permeability of Brucella outer membranes to hydrophobic substances (16), an interpretation in agreement with the fact that the outer membranes of $N$. meningitidis and $N$. gonorrhoeae, the other gram-negative bacteria affected by SPS, are also permeable to such substances (15). However, the anticoagulant, antiphagocytic, anticomplementary, and aminoglycoside-inactivating activities of SPS $(3,19,29)$ make it an essential component of most commercial blood culture media (19). Therefore, a substance with properties similar to those of SPS but that is nontoxic should be included in broths designed for hemocultures suspected of containing Brucella spp.

In summary, although further studies are necessary to assess the practical value of the proposed modifications, the use of pyruvate, a moderately acidic $\mathrm{pH}$, and an agent different from SPS are factors that should be taken into account in the improvement of growth media designed to detect Brucella spp. by $\mathrm{CO}_{2}$ release.

\section{ACKNOWLEDGMENTS}

We are grateful to Marisa Pardo for experimental assistance. This investigation was supported by Becton Dickinson-Spain and Becton Dickinson-Europe and by Fundación R. Areces. Fellowship support for A. Vitas from the Gobierno de Navarra is also acknowledged.

\section{REFERENCES}

1. Alton, G. G., L. M. Jones, and D. E. Pietz. 1976. Las técnicas de laboratorio en la brucelosis, 2nd ed. World Health Organization, Geneva.

2. Anhalt, J. P. 1978. New experimental approaches to detection of bacteremia, p. 109-143. In J. A. Washington II (ed.), The detection of septicemia. CRC Press, Inc., Boca Raton, Fla.

3. Belding, M. E., and S. J. Klebanofi. 1972. Effect of sodium polyanethol sulfonate on antimicrobial systems in blood. Appl. Microbiol. 24:691-698.

4. Chase, W. M. 1968. Buffers, p. 398. In C. A. Williams and M. W. Chase (ed.), Methods in immunology and immunochemistry, vol. II, appendix 2. Academic Press, Inc., New York.

5. Daley, C., I. Lim, J. Mora, and I. Wilkinson. 1990. Comparative evaluation of nonradiometric BACTEC and improved Oxoid Signal Blood Culture in a clinical laboratory. J. Clin. Microbiol. 28:1586-1590.

6. Díaz, R., and I. Moriyón. 1989. Laboratory techniques in the diagnosis of human brucellosis, p. 73-83. In E. J. Young and M. J. Corbel (ed.), Brucellosis: clinical and laboratory aspects. CRC Press, Inc., Boca Raton, Fla.

7. Eng, J. 1975. Effect of sodium polyanethol sulfonate in blood cultures. J. Clin. Microbiol. 1:119-123.

8. Gerhardt, P., D. R. MacGregor, A. G. Marr, C. B. Olsen, and J. B. Wilson. 1953. The metabolism of brucellae: the role of cellular permeability. J. Bacteriol. 65:581-586.

9. Graves, M. H., J. A. Morello, and F. E. Kocka. 1974. Sodium polyanethol sulfonate sensitivity of anaerobic cocci. Appl. Microbiol. 27:1131-1133.

10. Hauschald, A. H. W., and H. Pivnick. 1961. Continuous culture of Brucella abortus S-19. Can. J. Microbiol. 7:491-505.

11. Kelly, M. T., F. J. Roberts, D. Henry, I. Geere, and J. A. Smith. 1990. Clinical comparison of Isolator and BACTEC 660 Resin Media for blood culture. J. Clin. Microbiol. 28:1925-1927.

12. Kruse, R. H., W. H. Puckett, and H. Richardson. 1991. Biological safety cabinetry. Clin. Microbiol. Rev. 4:207-241.

13. Lambe, D. W., A. M. McPhedron, J. A. Mertz, and P. Stewart. 1973. Streptobacillus moniliformis isolated from a case of Haverhill fever: biochemical characterization and inhibitory effect of sodium polyanethol sulfonate. Am. J. Clin. Pathol. 60:854 860 .
14. López-Goñi, I., I. Moriyón, and J. B. Neilands. 1992. Identification of 2,3-dihydroxybenzoic acid as a Brucella abortus siderophore. Infect. Immun. 60:4496-4503.

15. Lysko, P. G., and S. A. Morse. 1981. Neisseria gonorrhoeae cell envelope: permeability to hydrophobic molecules. J. Bacteriol. 145:946-952.

16. Martínez de Tejada, G., and I. Moriyón. 1993. The outer membranes of Brucella spp. are not barriers to hydrophobic permeants. J. Bacteriol. 175:5273-5275.

17. McCabe, W. R., and G. G. Jackson. 1962. Gram negative bacteremia. Arch. Intern. Med. 110:856-864.

18. Reimer, L. G., and L. B. Reller. 1985. Effect of sodium polyanethol sulfonate and gelatin on the recovery of Gardnerella vaginalis from blood culture media. J. Clin. Microbiol. 21:686-688.

19. Reller, L. B., P. R. Murray, and J. D. MacLowery. 1982. Cumitech 1A, Blood cultures II. Coordinating ed., J. A. Washington II. American Society for Microbiology, Washington, D.C.

20. Rest, R. F., and D. C. Robertson. 1975. Characterization of the electron transport system in Brucella abortus. J. Bacteriol. 122:139-144.

21. Robertson, D. C., and W. G. McCullough. 1968. The glucose catabolism of the genus Brucella. I. Evaluation of pathways. Arch. Biochem. Biophys. 127:263-273.

22. Robertson, D. C., and W. G. McCullough. 1968. The glucose catabolism of the genus Brucella. II. Cell-free studies with $B$. abortus (S-19). Arch. Biochem. Biophys. 127:445-456.

23. Rodríguez-Torres, A., J. Fermoso, and R. Landínez. 1983. Brucelosis. Medicine (Spanish Ed.) 48:3126-3136.

24. Schnur, E. R., P. H. Azimi, and D. A. Belchis. 1989. Poor performance of BACTEC NR730 blood culture system in early detection of Neisseria meningitidis. J. Clin. Microbiol. 27:654 656.

25. Serrano, M. L., J. Llosa, C. Castells, J. Mendoza, J. M. Navarro, and M. de la Rosa. 1987. Detección radiométrica de bacteremias por Brucella. Enf. Infec. Microbiol. Clin. 5:139_ 142.

26. Sperry, J. A., and D. C. Robertson. 1975. Erythritol catabolism by Brucella abortus. J. Bacteriol. 121:619-630.

27. Sperry, J. A., and D. C. Robertson. 1975. Inhibition of growth by erythritol catabolism in Brucella abortus. J. Bacteriol. 124: 391-397.

28. Staneck, J. L., and S. Vincent. 1981. Inhibition of Neisseria gonorrhoeae by sodium polyanethol sulfonate. J. Clin. Microbiol. 13:463-467.

29. Traub, W. H., and B. L. Lowrance. 1970. Anticomplementary, anticoagulatory, and serum-protein precipitating activity of sodium polyanethol sulfonate. Appl. Microbiol. 20:465-468.

30. Vitas, A., I. Moriyón, R. Díaz, and C. Gamazo. 1993. Modification of the medium for BACTEC NR730 to increase $\mathrm{CO}_{2}$ released by Brucella, abstr. 670, p. 182. Abstr. 6th European Congress of Clinical Microbiology and Infectious Diseases. 1983, Seville, Spain.

31. Weinstein, M. P., S. Mirrett, L. G. Reimer, and L. B. Reller. 1990. Effect of altered headspace atmosphere on yield and speed of detection of the Oxoid Signal Blood Culture system versus the BACTEC radiometric system. J. Clin. Microbiol. 28:795797.

32. Wilson, J. B., and B. L. Dasinger. 1960. Biochemical properties of virulent and avirulent strains of Brucellae. Ann. N.Y. Acad. Sci. 88:1155-1166.

33. Wilson, M. L., M. P. Weinstein, L. G. Reimer, S. Mirrett, and L. B. Reller. 1992. Controlled comparison of the BacT/Alert and BACTEC 660/730 nonradiometric blood culture systems. J. Clin. Microbiol. 30:323-329.

34. Zimmerman, S. J., S. Gillikin, N. Sofat, W. R. Bartholomew, and D. Amsterdam. 1990. Case report and seeded blood culture study of Brucella bacteremia. J. Clin. Microbiol. 28:2139-2141.

35. Zobell, C. E., and K. F. Meyer. 1932. Metabolism studies on the Brucella group. J. Infect. Dis. 26:344-360. 